\title{
Vacuum-ultraviolet resonant photoabsorption imaging of laser produced plasmas
}

\author{
J. S. Hirsch, O. Meighan, J-P. Mosnier, P. van Kampen, W. W. Whitty, \\ and J. T. Costello ${ }^{\text {a) }}$ \\ School of Physical Sciences, and National Centre for Plasma Science and Technology, \\ Dublin City University, Dublin 9, Ireland \\ C. L. S. Lewis and A. G. MacPhee \\ School of Mathematics and Physics, Queens University of Belfast, Belfast BT7 1NN, Northern Ireland \\ G. J. Hirst, J. Westhall, and W. Shaikh \\ Central Laser Facility, Rutherford Appleton Laboratory, Chilton, Didcot OX11 OQX, England
}

(Received 6 June 2000; accepted for publication 7 August 2000)

\begin{abstract}
We present results from a vacuum-ultraviolet (VUV) "photoabsorption imaging" technique based on the measurement of the time and space resolved absorption of a quasimonochromatic VUV beam from a laser plasma light source. The use of VUV radiation as a probe beam permits direct access to resonance lines of (singly and more highly charged) ions and also to the resonant and nonresonant continua of atoms and ions. In this experiment we have confined ourselves to measurements using the $3 p-3 d$ resonances of $\mathrm{Ca}, \mathrm{Ca}^{+}$, and $\mathrm{Ca}^{2+}$ as markers of the temporal and spatial distribution of ground state atoms and ions in an expanding laser plasma plume. We show how time resolved column density maps may be extracted from such images. In addition we have extracted plasma plume velocities from the data, which compare well with an analytical laser ablation model.

(C) 2000 American Institute of Physics. [S0021-8979(00)07721-5]
\end{abstract}

\section{INTRODUCTION}

The period covering the mid-1960s to the early 1970s was one of intense research activity on plasmas produced with small-scale laser systems delivering pulses of less than a few Joules on the nanosecond timescale. A barrage of established and developed/adapted optical and particle diagnostic techniques were employed in the study of these plasmas. ${ }^{1,2}$ The 1970s and 1980s witnessed a shift in emphasis to research with large-scale laser systems designed to support fusion energy generation ${ }^{3}$ and x-ray laser programs. ${ }^{4}$ However, during the 1990s there was a marked return to the study of plasmas created with tabletop lasers. On the one hand this interest derives from a desire to develop compact, ultrashort pulse, soft x-ray light sources ${ }^{5}$ for lithography/ microscopy ${ }^{6}$ and spectroscopy. ${ }^{7}$ At the other end of the laser pulse duration scale, nanosecond laser systems are being extensively used for laser ablation/deposition studies. ${ }^{8,9}$

Direct imaging of the light emitted from plasma plumes with gated charge coupled device $(\mathrm{CCD})$ cameras $^{10,11}$ and also of the light absorbed from wavelength tuned lasers ${ }^{12}$ are increasingly important diagnostics of laser ablated/plasma plumes. The former provides space and time resolved information on excited states of atomic and molecular species, while the latter can provide corresponding data on ground state or "dark" species. However, tabletop tuneable lasers are limited for many practical purposes to wavelengths in excess of $300 \mathrm{~nm}$ if one requires pulse energies above a few $\mathrm{mJ}$. Hence they can access the resonance lines of at most

\footnotetext{
a)Electronic mail: jtc@physics.dcu.ie
}

singly charged atomic ions and even then not all such species.

A well established and extremely versatile technique for measuring photoabsorption spectra of almost all atoms and singly/multiply charged ions is the dual laser plasma (DLP) method. ${ }^{13-15}$ In DLP spectroscopic experiments, one laser plasma constitutes the absorbing "sample" while the other forms the vacuum ultraviolet (VUV) (continuum) backlighting source. ${ }^{16}$ VUV light passing through the sample plasma is dispersed by a spectrometer and recorded on a VUV sensitive array detector. ${ }^{17}$ Varying the delay between the formation of the sample and light source plasmas one can measure the time resolved photoabsorption spectrum of the sample plasma. By recording photoabsorption spectra in different spatial zones of the sample plasma it is possible to build up a picture of how the plasma evolves in space and time. Unfortunately use of the conventional DLP technique as a plasma diagnostic is very limited due to the necessity to probe sequentially the different plasma zones. The full spatial history of the plasma evolution must therefore be built up over many shots.

We show here that probing a laser ablated/plasma plume with quasimonochromatic VUV light can overcome this limitation. In contrast to the usual DLP spectroscopy experiment we pass a VUV beam, tuned to an atomic or ionic resonance, through a laser-plasma plume and measure the resultant transmitted image (or shadow). In this way we are able to obtain directly the spatial-temporal distribution of plasma species. This method has a number of attractive features for application to laser ablated/plasma plume characterization, namely: 
(i) VUV light can access resonance lines of all atoms and moderately charged ions. Hence one can track ions of any element and in any charge state with resonance energies up to the photon energy limit of the VUV source and any associated optics. In the current setup, the upper photon energy limit is $35 \mathrm{eV}$ since we are using near normal incidence optics.

(ii) VUV light can access the higher density regimes that are excluded from visible light photoabsorption/ shadowgraphy techniques.

(iii) The pulsed laser plasma light source emits VUV radiation typically for $1-50 \mathrm{~ns}$, depending on the heating pulse duration; hence the frame time or temporal resolution available can be short and achieved automatically without any additional fast switching requirement. By varying the interlaser delay the plasma may be shadowgraphed at different times after its creation.

However, the most important point is that the laser plasma source makes analysis of the transmitted light distribution relatively uncomplicated; it is, to a very good approximation, a direct image of photoabsorption within the plume. The main reasons for this are:

(i) The dimension and effective relative bandwidth of the laser plasma source means that it is essentially an incoherent source and hence image analysis is not complicated by the presence of interference patterns.

(ii) Refraction of a VUV beam in a plasma with given density gradient is significantly reduced compared to the case for a visible beam, with beam deviation angles scaling approximately as $\left(\lambda_{\text {probe }}\right)^{-2}$.

The use of VUV radiation as a probe has another very important advantage - it can be used to photoionize atoms or ions, a process immune from the usual radiation transfer problems, which complicate optical probing of plasmas with ground state (bound) resonance transitions. In particular, tuneable VUV radiation can be used to induce resonant photoionization in, e.g., $\mathrm{Ca}^{+}$(Ref. 18) via the path

$$
\begin{aligned}
\mathrm{Ca}^{+}\left(3 p^{6} 4 s^{2} S\right)+h \nu & \rightarrow \mathrm{Ca}^{+*}\left(3 p^{5} 4 s 3 d^{2} P\right) \\
& \rightarrow \mathrm{Ca}^{2+}\left(3 p^{6} S\right)+e^{-} .
\end{aligned}
$$

The branching ratio for fluorescence to electron emission processes for such VUV excited (inner-shell) resonances, tends to be significantly less than $10^{-4}$. Hence, almost all absorbed photons are converted to electrons and one does not have to be concerned about multiple photon absorption/reemission cycles in the plasma plume. Thus a simple BeerLambert type law

$$
I(E)=I_{0}(E) \exp \left[-\sigma(E) \int n(l) d l\right],
$$

where $N L=\int n(l) d l$ is the column density of the species of interest, may be applied to each individual pixel on an array detector. A correction for the magnification due to beam divergence yields a cross-sectional view of photoabsorption within the plasma plume. For those atoms (and ions) for which measured photoionization cross sections are available one can extract column density values $(N L)$ or (for well-

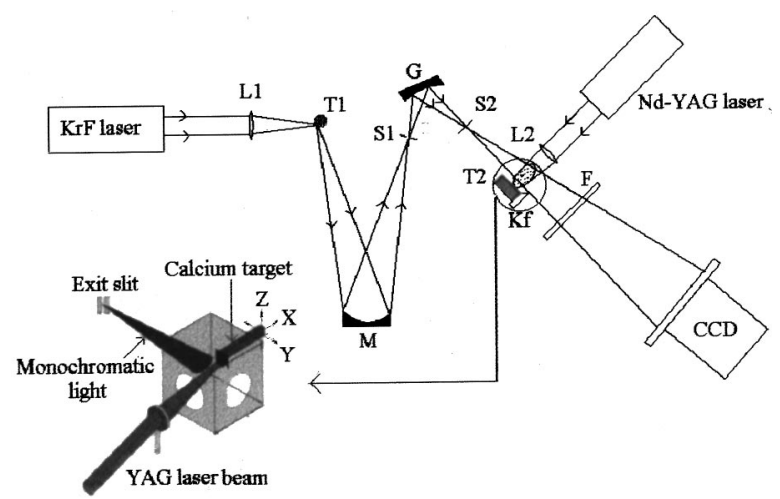

FIG. 1. Schematic of the experimental set up: $(G)$ grating $1200 \mathrm{~g} / \mathrm{mm},(L 1)$ fused silica lens $100 \mathrm{~mm}$ focal length, (L2) cylindrical lens $150 \mathrm{~mm}$ focal length, ( $T 1)$ tungsten target, (T2) calcium target, $(S 1 / S 2)$ monochromator entrance/exit slit, $(F)$ aluminum filter, $(\mathrm{Kf})$ knife edge, $(M)$ iridium coated $\operatorname{mirror}(f=1 \mathrm{~m})$.

defined plume dimensions) actual atomic or ionic number densities $(N)$. Since peak values of photoionization cross sections for resonant inner-shell excitations can be as high as 2200 Mbar $\left(\mathrm{Ca}^{+}\right)$, column densities down to $\sim 5$ $\times 10^{13} \mathrm{~cm}^{-2}$ may be measured. The use of inner shell excitations brings another advantage; excitation energies for different stages of ionization tend to be displaced slightly from each other. Thus, keeping all other experimental conditions identical, one can readily image different ionization stages of the same atom in one experiment by tuning the monochromator energy to the relevant resonance lines.

We have chosen to showcase this technique by imaging the temporal and spatial distribution of the ground state of $\mathrm{Ca}, \mathrm{Ca}^{+}$, and $\mathrm{Ca}^{2+}$ in a laser plasma plume using the $3 p$ $-3 d$ resonance line for each species. In particular, $\mathrm{Ca}^{+}$was chosen since its absolute VUV cross section has been measure ${ }^{19}$ which permitted us to construct a column density map of $\mathrm{Ca}^{+}$in the plasma plume. We have also been able to track in time and extract the expansion velocity of the $\mathrm{Ca}^{+}$ component of the plume, which turns out to be in good agreement with the model of Singh and Narayan. ${ }^{20}$ In addition we have measured a charge state on the $3 p^{6}\left({ }^{1} S\right)$ $\rightarrow 3 p^{5} 3 d\left({ }^{1} P\right)$ resonance of $\mathrm{Ca}^{2+}$, which is accessible only with this VUV method.

\section{EXPERIMENT}

A schematic of the experiment is shown in Fig. 1. A tungsten plasma, generated by a $\mathrm{KrF}$ excimer laser $(248 \mathrm{~nm}$, $0.17 \mathrm{~J}, 20 \mathrm{~ns}$ ) focused to a $\sim 100 \mu \mathrm{m}$ point by a $10 \mathrm{~cm}$ focal length $f / 5$ fused silica lens, acted as a VUV continuum light. This point plasma was located $1 \mathrm{~m}$ from a $f / 10$ iridium coated mirror of focal length $0.5 \mathrm{~m}$ with which it made an angle of incidence of $7.5^{\circ}$. The plasma source was imaged with unit magnification onto the entrance slit of a $0.2 \mathrm{~m}$ focal length vacuum $f / 4.5$ monochromator (Acton ${ }^{\mathrm{TM}}$ VM-502). Operating with an exit slit width of $250 \mu \mathrm{m}$ it yielded a bandwidth of $\sim 0.9 \mathrm{eV}$, which has implications for the interpretation of the shadowgrams presented in the Sec. III which 
will be discussed there. Due to the near normal angle of incidence on the focusing mirror, we observed no plasma radiation with a photon energy $>35 \mathrm{eV}$.

The VUV pulse duration was measured by placing a plastic scintillator (NE102 $\mathrm{A}^{\mathrm{TM}}$ film of thickness $0.1 \mathrm{~mm}$ ) at the exit slit of the monochromator and detecting the fluorescence signal with a fast photomultiplier tube coupled to a digital storage oscilloscope. The response time of the system was better than $5 \mathrm{~ns}$. The VUV pulse duration was observed to be $50 \pm 5 \mathrm{~ns}$ in the $20-35 \mathrm{eV}$ photon energy range rising to $60 \pm 5 \mathrm{~ns}$ at $10 \mathrm{eV}$ and $100 \pm 5 \mathrm{~ns}$ at $5 \mathrm{eV}$.

VUV radiation emanating from the exit slit was directed through a calcium plasma produced by a Nd-YAG laser $(1064 \mathrm{~nm}, 0.3 \mathrm{~J}, 10 \mathrm{~ns})$. The Nd-Yag laser was synchronized to the $\mathrm{KrF}$ laser pulse and focused to a horizontal line of length $8 \mathrm{~mm}$ and width $\sim 0.25 \mathrm{~mm}$ by a $15 \mathrm{~cm}$ focal length cylindrical lens. The on-target irradiance was $\sim 5$ $\times 10^{8} \mathrm{~W} / \mathrm{cm}^{2}$ which yields, according to the model of Colombant and Tonon, ${ }^{21}$ an estimated equilibrium plasma temperature of $\sim 3.5 \mathrm{eV}$ and an initial dominant charge state of triply ionized $\mathrm{Ca}$. A continuously variable delay in the range of nanoseconds to tens of microseconds with a jitter of $<5 \mathrm{~ns}$ was inserted between the Nd-YAG and $\mathrm{KrF}$ laser pulses. In order to shield the detector from both scattered $248 \mathrm{~nm}$ laser light and direct calcium plasma emission, a $0.4 \mu \mathrm{m}$ thick aluminum filter was placed in front of the CCD camera. When combined with the near normal incidence iridium coated mirror, the composite filter restricted the monochromator transmission to light with a photon energy between 16 and $35 \mathrm{eV}$. In addition, a knife edge (Kf in Fig. 1) was located close to the calcium target. It protruded some 200 $\mu \mathrm{m}$ outwards from the surface of the calcium target in order to occlude the brightest part of the calcium plasma from the CCD. In this way we were able to further reduce the background signal on the CCD due to calcium plasma emission. It also provided a well-defined edge on the images and protected the aluminum filter, which was easily damaged by plasma debris in its absence.

The VUV shadowgrams were recorded on a $2048 \times 1024$ pixel ANDOR $^{\mathrm{TM}}$ CCD camera with a pixel size of 13 $\mu \mathrm{m} \times 13 \mu \mathrm{m}$, placed $50 \mathrm{~cm}$ from the exit slit of the monochromator and $\sim 38 \mathrm{~cm}$ from the calcium plasma. In practice we found that the typical source flux was only 20 counts/ pixel and hence all images were recorded using "superpixels" of $8 \times 8$ pixel size in order to improve the image signal to noise ratio. Since these superpixels yielded an effective pixel size $\sim 100 \mu \mathrm{m} \times 100 \mu \mathrm{m}$, the spatial sampling frequency of the CCD was reduced to 10 samples $/ \mathrm{mm}$ for all images shown here.

The procedure we followed was to record an image of the background signal without firing any laser. The $\mathrm{KrF}$ laser was fired and an image of the continuum was recorded with background subtraction $\left[\int I_{0}(E) d E\right]$, where $I_{0}(E) d E$ is the VUV fluence $\left(\mathrm{J} \mathrm{cm}^{-2}\right)$ between $E$ and $E+d E$ falling on each pixel. A calcium plasma was created and an image of any residual VUV emission from it was saved as a background signal. Finally both lasers were fired at a set time delay and a photoabsorption image was recorded with background and front plasma emission subtraction $\left[\int I(E) d E\right]$ where
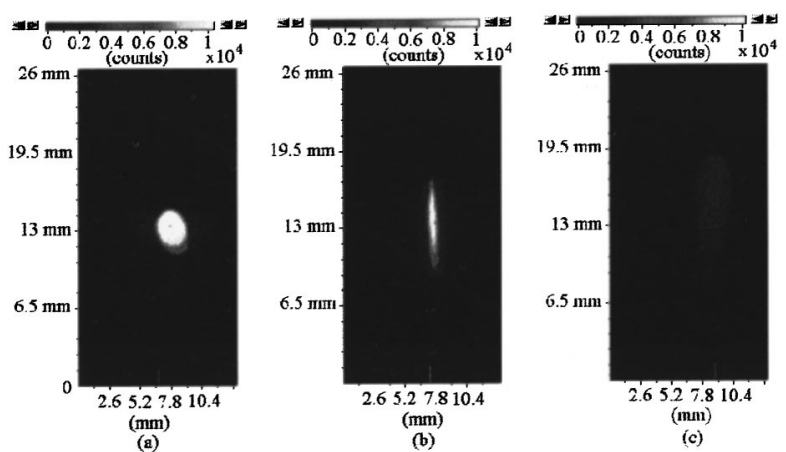

FIG. 2. Footprint of the VUV [33.2 eV beam at (a) $11.5 \mathrm{~cm}$, (b) $28 \mathrm{~cm}$, and (c) $50 \mathrm{~cm}$ ] from exit slit. The ordinate represents distance in $\mathrm{mm}$ along the horizontal direction, i.e., perpendicular to the long dimension of the (vertical) exit slit.

$I(E) d E$ represents the transmitted VUV fluence $\left(\mathrm{J} \mathrm{cm}^{-2}\right)$ between $E$ and $E+d E$. The spatially resolved absorbance was obtained by evaluating

$$
A=\log _{10}\left(\frac{\int I_{0}(E) d E}{\int I(E) d E}\right) .
$$

\section{RESULTS}

The "footprint" of the VUV beam emerging from the monochromator was measured at different distances (11.5, 28 , and $50 \mathrm{~cm}$ ) from the exit slit. The resulting images are shown in Fig. 2. The dimensions (full widths and heights) are $3.8 \mathrm{~mm} \times 2.6 \mathrm{~mm}$ at $11.5 \mathrm{~cm}, 9.1 \mathrm{~mm} \times 0.8 \mathrm{~mm}$ at $28 \mathrm{~cm}$, and $\sim 15 \mathrm{~mm} \times 2.7 \mathrm{~mm}$ at $50 \mathrm{~cm}$. The calcium target was located at the $11.5 \mathrm{~cm}$ position for all photoabsorption measurements and hence the area of plasma sampled was $\sim 3.8$ $\mathrm{mm} \times 2.6 \mathrm{~mm}$. The uncertainty in the image width at the 50 $\mathrm{cm}$ position is due to the fact that the image is cut off by the $\mathrm{Al}$ thin film filter holder and hence we are forced to estimate this number from the extrapolated image profile. It is clear that the VUV beam diverges in the horizontal plane with an angle of $\sim 0.03 \mathrm{rad}$. In the vertical plane it is first brought to a horizontal focus (extrapolated to lie $\sim 31 \mathrm{~cm}$ from the exit slit) and then diverges with an angle of $\sim 0.014 \mathrm{rad}$.

To measure the magnification of the system in the horizontal plane we used a moveable knife edge as shown in Fig. 1. Starting at the cutoff position (i.e., blocking all VUV radiation from the backlighter) the knife edge was retracted from the VUV beam in steps of $200 \mu \mathrm{m}$ and the corresponding change in image size recorded. The results yielded a value of $3.4 \pm 0.1$ for the horizontal magnification. The magnification in the vertical plane was also measured using a moveable knife edge and a value of $1.0 \pm 0.1$ obtained. In addition we were able to use the same knife edge data to estimate the horizontal and vertical resolution of the system (see Fig. 3). From the shape of the knife edge traces in both the vertical and horizontal directions we measured values of $0.25 \mathrm{~mm}$ in the horizontal direction and $0.30 \mathrm{~mm}$ in the vertical direction. We have taken the distance over which the intensity drops from $90 \%$ to $10 \%$ in order to arrive at these values of total system spatial resolution. These numbers perhaps need some explanation at this point. Since the VUV 


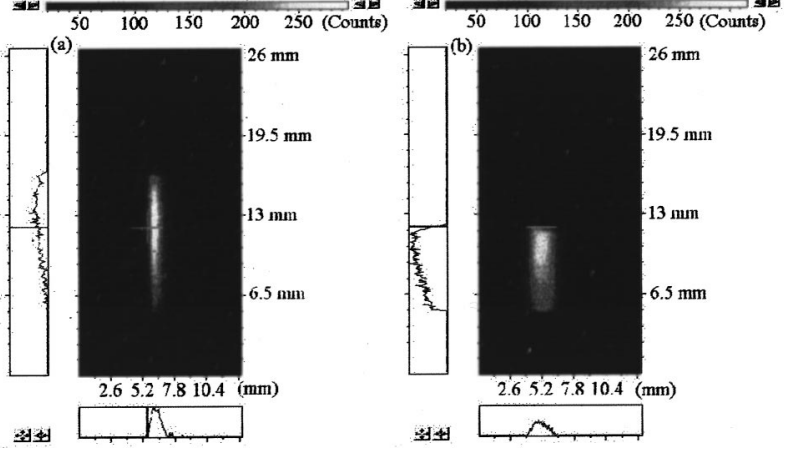

FIG. 3. Typical shadowgrams used for the determination of the: (a) vertical and (b) horizontal spatial resolution of the system.

light is first brought to a secondary focus before diverging and impinging on the CCD, one obtains "automatic" vertical spatial resolution determined mainly by the superpixel dimensions. From Nyquist the spatial frequency limit of the detector becomes $(2 \times \text { superpixel size })^{-1}=5$ linepairs $/ \mathrm{mm}$ or $(0.2 \mathrm{~mm})$. The measured value $0.25 \mathrm{~mm}$ is slightly higher and the difference reflects the need to include an additional contribution due residual spectrometer-optics aberrations.

In the orthogonal plane the beam simply diverges from the $250 \mu \mathrm{m}$ wide slit. Simple ray tracing shows that the width of the beam subtended by any one superpixel at the sample position is $>200 \mu \mathrm{m}$. Convolving a $250 \mu \mathrm{m}$ slit with a $100 \mu \mathrm{m}$ pixel size will yield a spatial resolution of $\sim 0.35$ $\mathrm{mm}$ (FWHM of a trapezoid instrument function) which is close to the measured value. In addition we should also mention that the plasma expansion during the $50 \mathrm{~ns}$ VUV beam duration gives rise to dynamic smearing on the shadowgrams. For a lower limit on the plume expansion velocity of $\sim 10^{5} \mathrm{~cm} \mathrm{~s}^{-1}$ this amounts to a minimum value of $\sim 50 \mu \mathrm{m}$. Further, the length of the plume is $8 \mathrm{~mm}$ along the line of sight of the VUV beam. Hence, the vertical and horizontal divergences of 14 and 30 mrad lead to smearing over a distance of 110 and $240 \mu \mathrm{m}$, respectively.

In Fig. 4(a) we show time resolved absorption images (shadowgrams) recorded by tuning the monochromator to the $3 p-3 d$ resonances line of atomic $\mathrm{Ca}$ at $31.4 \mathrm{eV}$. The data are absorbance distributions and reflect the spatial distribution of ground state $\mathrm{Ca}$ in the plasma plume at times of 100 ns $-3 \mu$ s after calcium plasma generation. The sharp edge at the top of each image is the shadow of the knife edge located close to the Ca target (Fig. 1) while the sharp cutoff at the bottom of the image is due to the edge of the thin film aluminum holder. An area of intense absorption (and hence atomic $\mathrm{Ca}$ concentration) remains close to the surface of the $\mathrm{Ca}$ target until approximately $1 \mu$ s at which point the $\mathrm{Ca}$ atoms have expanded outward to a dimension comparable to the horizontal extent of the VUV beam. For time delays in excess of $1 \mu$ s the most dense region of $\mathrm{Ca}$ atoms in the plume becomes detached from the target surface and overall the absorption decreases but even at $3 \mu$ s there is still a measurable absorption signal. The apparent region of low absorption at the bottom of all the images shown in the article is an artifact resulting from damage to the Al-VUV filter.
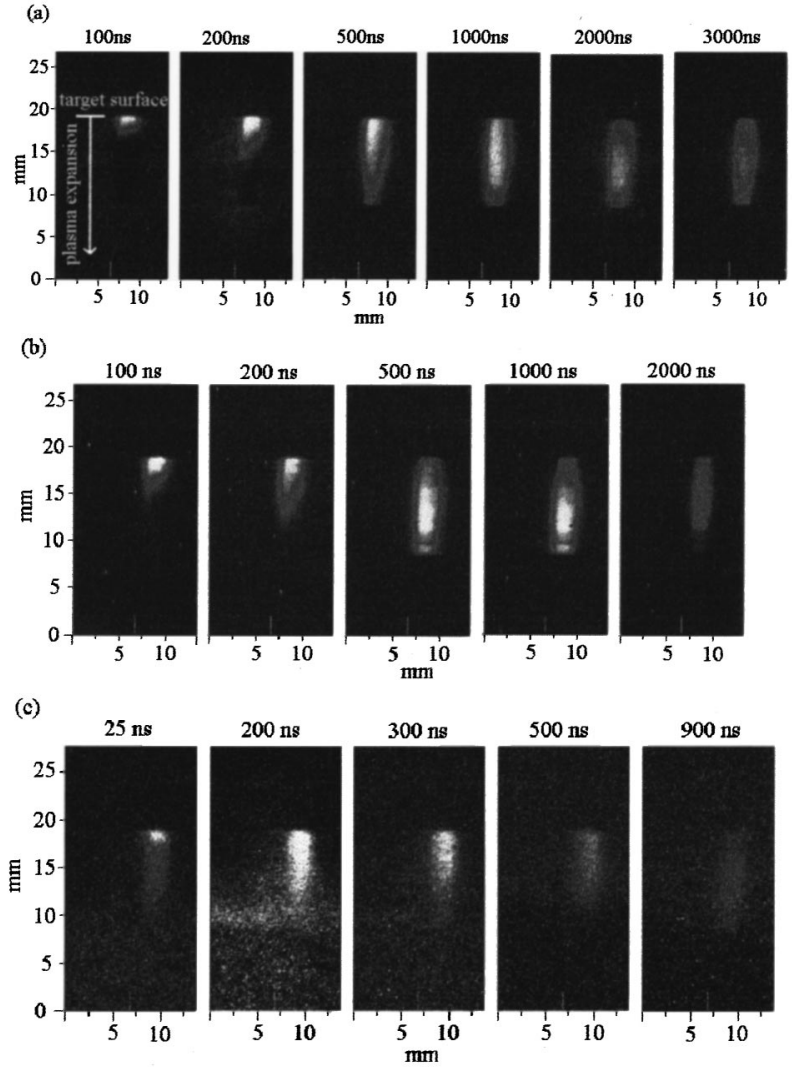

FIG. 4. (a) Atomic calcium absorbance spatial distribution using the $3 p-3 d$ resonance at $31.4 \mathrm{eV}$ for different time delays; (b) $\mathrm{Ca}^{+}$absorbance distribution using the $3 p-3 d$ resonance at $33.2 \mathrm{eV}$ for different time delays; (c) $\mathrm{Ca}^{2+}$ absorbance distribution using the $3 p-3 d$ resonance at $34.7 \mathrm{eV}$ for different time delays. The ordinate represents distance in $\mathrm{mm}$ normal to the calcium target along the direction of the incoming Nd-YAG laser beam.

In Fig. 4(b) we show time resolved absorbance distributions recorded at the $3 p-3 d(33.2 \mathrm{eV})$ resonance of $\mathrm{Ca}^{+}$for time delays ranging from $100 \mathrm{~ns}$ to $1 \mu \mathrm{s}$. Here again we observe a behavior similar to that of atomic calcium. However, it is clear that the hotter $\mathrm{Ca}^{+}$ions have a larger horizontal velocity component and as a consequence the plume expands more quickly and the $\mathrm{Ca}^{+}$absorption signal drops more rapidly than in the atomic calcium case.

In Fig. 4(c), we show VUV shadowgrams recorded at the $3 p\left({ }^{1} S\right)-3 d\left({ }^{1} P\right)$ resonance $(34.7 \mathrm{eV})$ of $\mathrm{Ca}^{2+}$ for time delays ranging from 25 to $900 \mathrm{~ns}$. These shadowgrams were recorded at the short wavelength limit of the near normal incidence focusing mirror where the continuum signal $I_{0}$ and the transmitted signal $I$ were both very weak. In addition, in the cases of $\mathrm{Ca}$ and $\mathrm{Ca}^{+}$the $3 p-3 d$ resonance linewidths are on the order of $100 \mathrm{meV}$ and hence they absorb a significant portion of the $0.9 \mathrm{eV}$ width VUV beam. For $\mathrm{Ca}^{2+}$, the $3 p-3 d$ resonance linewidth is much narrower $(\mathrm{meV})$ and hence only a small part of the VUV radiation is absorbed by these ions. As a result, there is only a small difference between the $I$ and $I_{0}$ signals. These effects resulted in poorer quality images. Moreover in this case we are dealing with a bound to bound transition and radiation transfer potentially becomes a consideration. Since there is no measured oscillator strength for this line we have calculated 


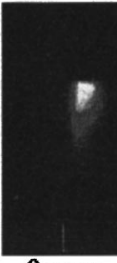

$0 \mathrm{~mm}$
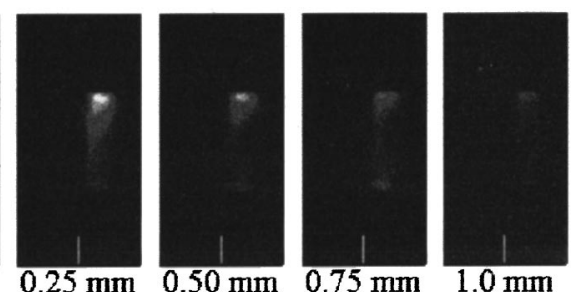

FIG. 5. Spatial scan of the calcium plasma plume at $200 \mathrm{~ns}$ time delay. The distances in $\mathrm{mm}$ represent the incremental steps of the calcium target translation.

it with the COWAN suite of $\operatorname{codes}^{22}$ which yielded a value of 1.4. Hence it is a strong transition and absorption measurements with it are open to interference from plasma opacity effects. However the $\mathrm{Ca}^{2+}$ shadowgram shows that it is possible to record multiply charged ion distributions and track their evolution in time. We will refine our experiment, further explore, and develop this aspect of the work in future experiments (see Sec. V).

\section{DISCUSSION}

\section{A. Ca plasma expansion velocities}

The recorded shadowgrams embody information concerning the size of the expanding plasma plume at different time delays and hence may be used to extract plasma expansion velocities. Figure 5 shows a spatial scan of the plasma plume in the horizontal plane at a $200 \mathrm{~ns}$ time delay for the $\mathrm{Ca}^{+} 3 p-3 d$ resonance line at $33.2 \mathrm{eV}$. The calcium target was translated from its initial position out of the continuum beam in $0.25 \mathrm{~mm}$ steps until no trace of the absorption signal remained. The absorption signal dropped to zero following translation of the calcium target by $1 \mathrm{~mm}$ on which we have to add $200 \mu \mathrm{m}$ due to the initial position of the target relative to the knife edge. Hence, we conclude that the size of the $\mathrm{Ca}^{+}$component of the plasma was $1.2 \pm 0.2 \mathrm{~mm}$ at $200 \mathrm{~ns}$. To check this result, the $\mathrm{Ca}^{+}$plume size was directly read off the shadowgram with the calcium target restored to its initial position. Knowing that the magnification of the system is 3.4 and that the dimension (full extent) of the plasma plume normal to the $\mathrm{Ca}^{+}$target on the shadowgram is 3.95 mm one again obtains $\sim 1.2 \mathrm{~mm}$ for the horizontal dimension in agreement with the knife edge measurements.

We have found that the intensity distribution of each image along an axis normal to the target is usually a slightly asymmetric bell shaped function. For our current purposes we take the FWHM of this distribution to represent the plasma dimension normal to the target surface In practice we used this measurement of the image full width at half maximum to estimate the velocity of the $\mathrm{Ca}^{+}$ions at defined time delays above $50 \mathrm{~ns}$. Based on uncertainties in measurements of the plume size, the projection magnification and the probing time, the velocity was estimated to an accuracy of $\pm 15 \%$.

These measurements are compared with calculations obtained by solving numerically ${ }^{23}$ the equation describing the adiabatic phase of the plasma expansion as illustrated in Fig. 6. The adiabatic expansion equation can be written as

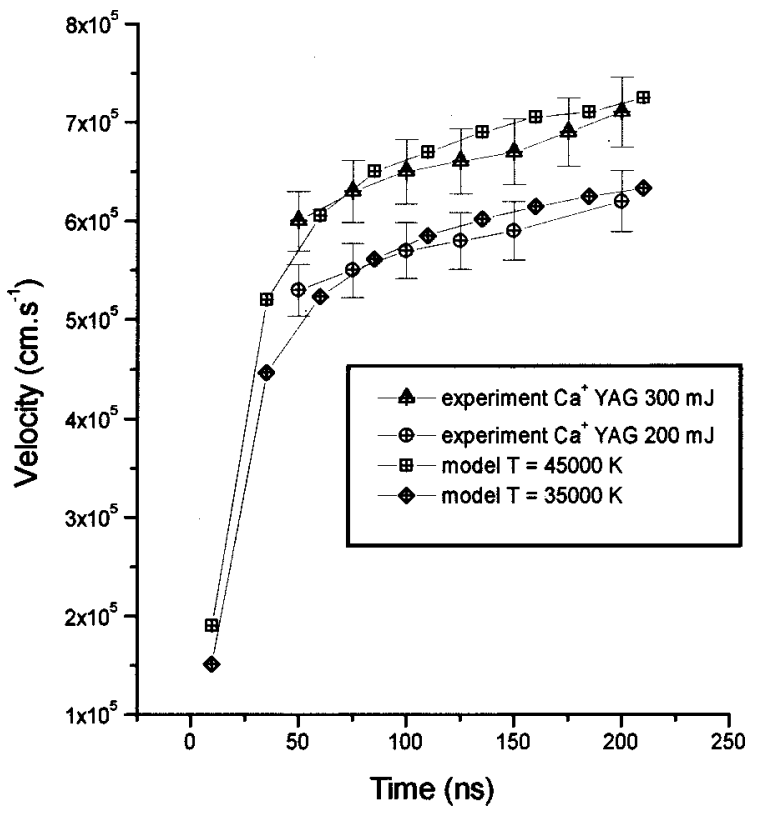

FIG. 6. Comparison of the measured $\mathrm{Ca}^{+}$ion expansion velocity with calculated whole plasma values obtained using the laser ablation model of Singh and Narayan (see Ref. 20).

$$
\begin{aligned}
X(t)\left[\frac{d^{2} X}{d t^{2}}\right] & =Y(t)\left[\frac{d^{2} Y}{d t^{2}}\right] \\
& =Z(t)\left[\frac{d^{2} Z}{d t^{2}}\right] \\
& =\frac{k T_{0}}{M}\left[\frac{X_{0} Y_{0} Z_{0}}{X(t) Y(t) Z(t)}\right],
\end{aligned}
$$

where $X_{0}, Y_{0}, Z_{0}$ are the initial orthogonal edge positions of the plasma after the termination of the laser pulse, $M$ is the atomic mass, $k$ is Boltzmann's constant, and $T_{0}$ is the isothermal temperature. The velocity calculations were performed using as initial spatial conditions a line plasma of size of $8 \mathrm{~mm} \times 0.25 \mathrm{~mm}$ with an initial velocity of $10^{5} \mathrm{~cm} \mathrm{~s}^{-1}$ for different plasma temperatures.

The measured velocities are in good agreement with the results of the theoretical model. The plasma velocity increases rather slowly with time going from $6 \times 10^{5} \mathrm{~cm} \mathrm{~s}^{-1}$ at $50 \mathrm{~ns}$ to $7.15 \times 10^{5} \mathrm{~cm} \mathrm{~s}^{-1}$ at $200 \mathrm{~ns}$. The effect of lowering the laser energy from 300 to $200 \mathrm{~mJ}$ is a reduction in plasma expansion velocity from $6 \times 10^{5}$ to $5.2 \times 10^{5} \mathrm{~cm} \mathrm{~s}^{-1}$ at $50 \mathrm{~ns}$ after calcium plasma initiation. Even though the model is designed to predict the motion of the entire plasma, it is interesting to see that there is good agreement between calculated plasma velocity and observed ground state $\mathrm{Ca}^{+}$velocity evolution. For a measured Nd-YAG laser pulse of energy $300 \mathrm{~mJ}$, a model plasma temperature of $45000 \mathrm{~K}$ yields a good fit. For a $200 \mathrm{~mJ}$ laser pulse we obtain a good agreement for a model plasma temperature of $35000 \mathrm{~K}$. These results are consistent with a plasma temperature dependence on the laser pulse energy scaling as $E_{\text {laser }}^{3 / 5}$. However since the laser pulse duration was fixed (10 ns) for these experiments as was the focal spot size, this behavior also indicates that 
the plasma temperature obeys the $T=a \Phi^{3 / 5}$ scaling law, where $\Phi$ is the laser irradiance on target $\left(\mathrm{W} / \mathrm{cm}^{2}\right)$.

\section{B. Column density map of $\mathrm{Ca}^{+}$}

To determine values of the column density $N L$, the equivalent width $W_{E}$ of the $3 p-3 d \mathrm{Ca}^{+}$absorption resonance line has to be determined. It can be found by inserting the shadowgram data values, i.e., $\int_{\Delta E} I_{0}(E) d E$ and $\int_{\Delta E} I(E) d E$ into the equation given by ${ }^{24}$

$$
W_{E}=\Delta E\left(\frac{\int_{\Delta E}\left[I_{0}(E)-I(E)\right] d E}{\int_{\Delta E} I_{0}(E) d E}\right)
$$

with

$$
\int I(E) d E=\int I_{0}(E) \exp [-\sigma(E) N L] d E .
$$

Using the numerical solution of Eq. (2) we can then associate a value of $N L$ with every value of $W_{E}$ found experimentally to create a map of column density within the plasma plume. In order to evaluate numerically expression (2) for any given $N L$ we use the measured experimental resonance photoionization cross section of $\mathrm{Ca}^{+} .{ }^{19}$ Since the reciprocal linear dispersion of the $0.2 \mathrm{~m}$ spectrometer was 4 $\mathrm{nm} / \mathrm{mm}$ and we were operating with a $250 \mu \mathrm{m}$ exit slit, the interval $\Delta E$ was $0.89 \mathrm{eV}$, which in turn sets the limits of the integration. Finally $I_{0}(E)$ is approximately constant over the interval $\Delta E$ and hence the expression for the equivalent width simplifies to

$$
W_{E}=\int[1-\exp [\sigma(E) N L]] d E .
$$

In particular cases, e.g., when dealing with an optically thin plasma, it can be shown that the equivalent width varies linearly with the product $N L$. In contrast, in the optically thick case, it can be shown to follow a square root dependence on $N L$. We can then express the equivalent width in these two specific cases.

(i) Optically thin plasma:

$$
W_{E}=\left(\frac{\pi e^{2}}{m c}\right) \times h \times f \times N L,
$$

(ii) optically thick plasma:

$$
W_{E}=\left(\frac{2 \pi e^{2} \times h \times f \times N L \times \Gamma}{m c}\right)^{1 / 2},
$$

where $m$ is the atom/ion mass, $c$ is the velocity of light, $h$ is the Planck constant, $f$ is the oscillator strength, and $\Gamma$ is the half width of a Lorentzian curve which best approximates the atomic line shape function. Figure 7 shows three plots of the equivalent width as a function of the product $N L$, for the $3 p-3 d$ transition of $\mathrm{Ca}^{+}$which has an oscillator strength $f=2.3$.

As expected, for small column density $(N L)$ values, $\left(N L<1 \times 10^{14}\right)$, the general expression of the equivalent width varies linearly with $N L$ in agreement with the optically thin case. However for larger values of $N L$, the optically thick approximation, although reasonable in the $(0.7-1.2)$ $\times 10^{15} \mathrm{~cm}^{-2}$ range, diverges quite dramatically at higher val-

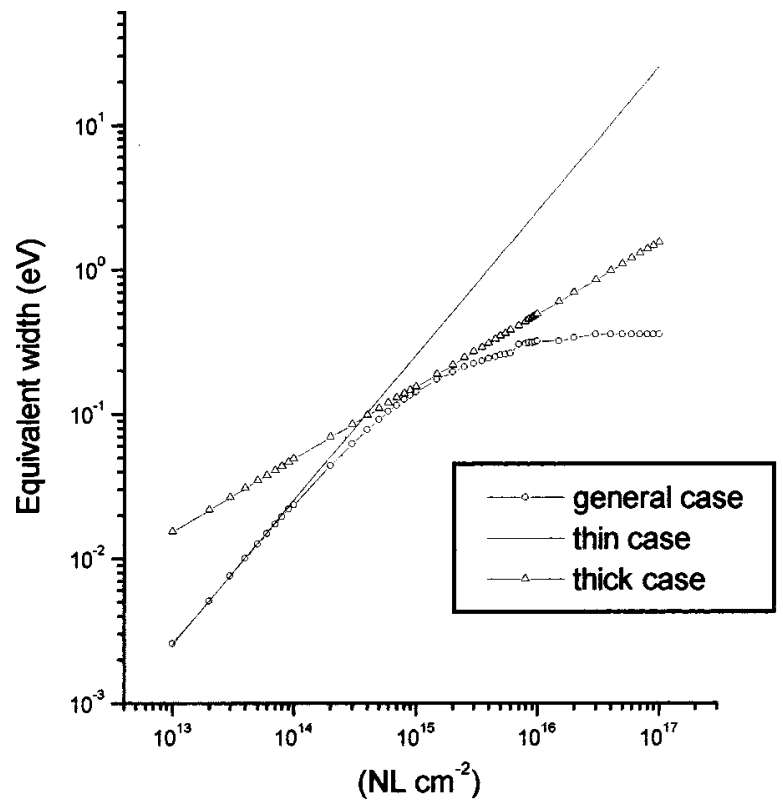

FIG. 7. Plot of the three different expressions of the equivalent width for the $\mathrm{Ca}^{+}(3 p-3 d)$ resonance line, i.e., general expression, optically thin case, and optically thick case.

ues of column density. For values of $N L>1.10^{16} \mathrm{~cm}^{-2}$ the curve tends to an asymptotic value of $0.35 \mathrm{eV}$. This behavior can be explained by noting that the $3 p-3 d \mathrm{Ca}^{+}$resonance at $33.2 \mathrm{eV}$ is very strong (with a peak cross section of 2200 Mbar), and the values obtained when solving $W_{E}=\int[1$ $-\exp [-\sigma(E) N L]] d E$ reach saturation for $N L>2.10^{15} \mathrm{~cm}^{-2}$. This is clear from Fig. 8 where we plot $1-\exp [-\sigma(E) N L]$ for different $N L$.

As a result, except for very long time delays ( $\Delta t$ $\geqslant 2500 \mathrm{~ns}$ ), shadowgrams display saturated absorption and values of equivalent width [obtained using Eq. (3)] $W_{E}$ are all $>0.3 \mathrm{eV}$. This is clear from Figs. 9(a) and 9(b). Hence we only extract column densities from the shadowgram taken at 2500 ns [Fig. 9(c)] and this is shown in Fig. 10. Since at this time delay the plume is now larger than the VUV beam footprint and also quite homogeneous we do not see any spatial variation in $N L$. Since we have the advantage of broad tuneability over a wide VUV photon energy range we

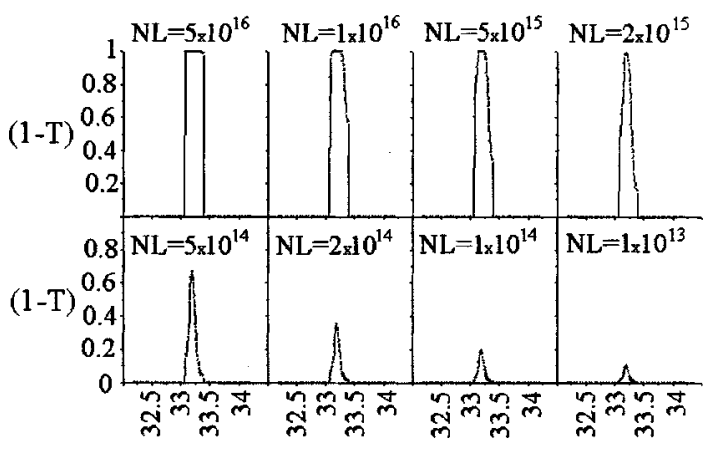

(eV)

FIG. 8. Plots of $1-T$, where $T$ is the transmission $[\exp (-\sigma(E) N L)]$ for the $\mathrm{Ca}^{+}(3 p-3 d)$ resonance line for different values of column density using the experimental $\sigma(E)$ of Lyons et al. (see Ref. 19). 


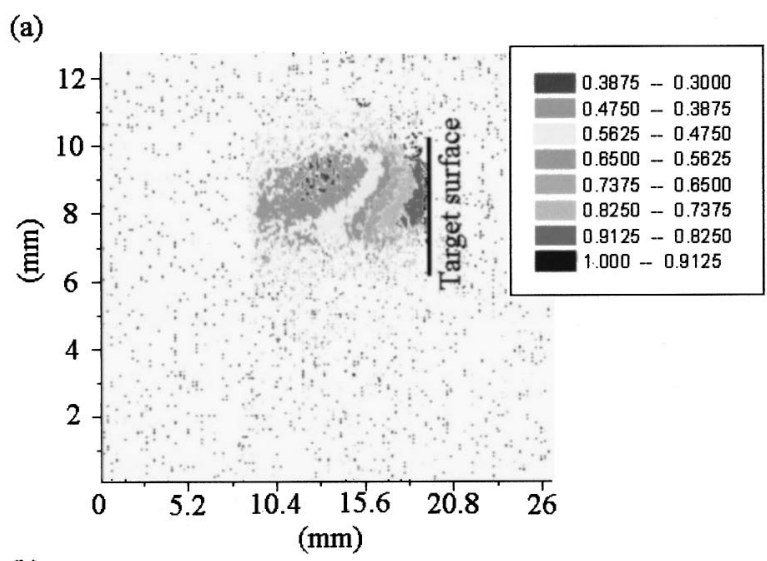

(b)

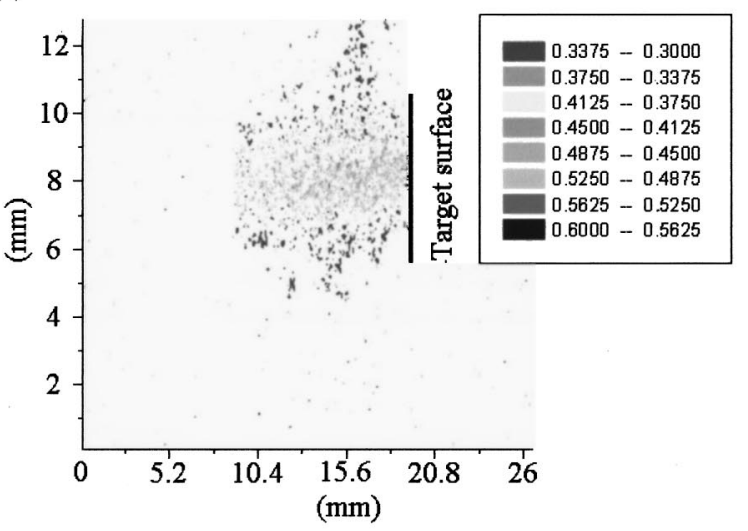

(c)

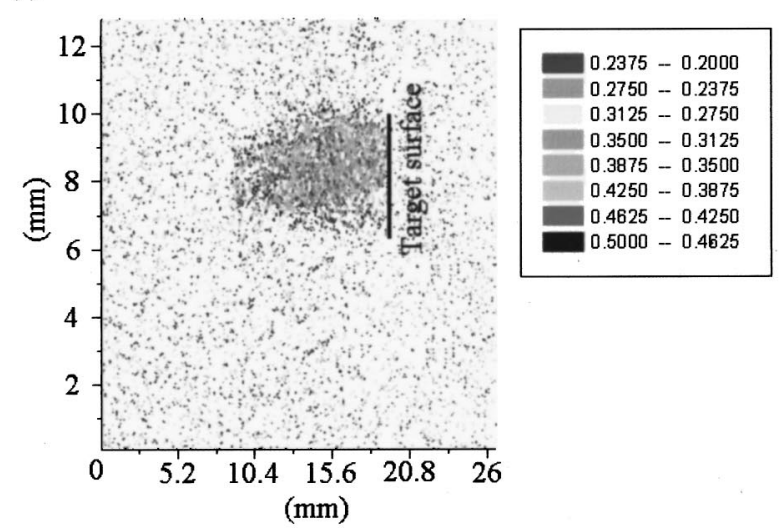

FIG. 9. Equivalent width distributions for the $\mathrm{Ca}^{+}(3 p-3 d)$ resonance at different time delays: (a) $200 \mathrm{~ns}$, (b) $1000 \mathrm{~ns}$ and, (c) $2500 \mathrm{~ns}$.

may choose from one of the many weaker $\mathrm{Ca}^{+}$resonances in order to probe higher ion-density regions of the plume. We will explore this option in future experiments.

\section{CONCLUSIONS}

We have recorded time and space resolved VUV absorbance distributions using the $3 p-3 d$ resonances of ground state $\mathrm{Ca}, \mathrm{Ca}^{+}$, and $\mathrm{Ca}^{2+}$. From these shadowgrams we have extracted values of the plasma plume size at different time delays and hence plasma expansion velocities. These results are in good agreement with computed plasma expansion velocity predictions. We have also shown that with appropriate

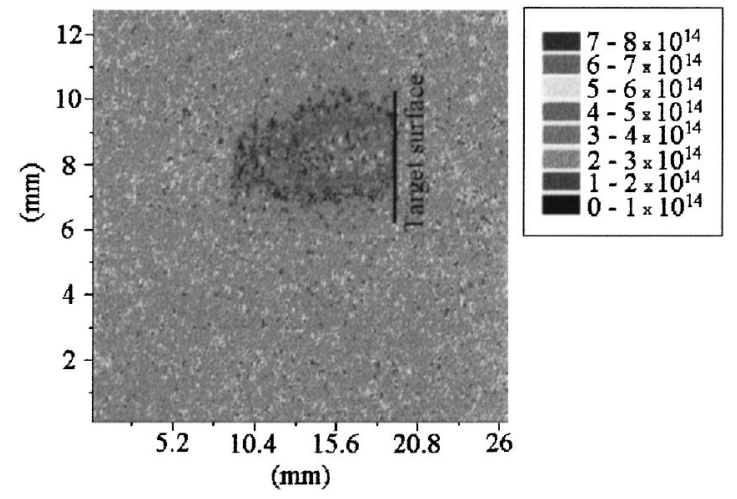

FIG. 10. Column density map for an interlaser time delay of 2500 ns corresponding to Fig. 9(c).

choice of inner-shell resonance it is possible to extract time resolved maps of column densities for ions from the recorded images.

Looking to the future, on the development of the basic technique one could also make measurements in the nonresonant part of the photoionization continuum. This has the advantage that the spectral resolution needed is significantly smaller than in the case of resonances. However, it has one further and very important advantage. The number of atoms and ions for which absolute photoionization cross sections are available is very small. However, the nonresonant part of the photoionization continuum cross section of all atoms and ions can be computed with an accuracy of better than $10 \%$. Hence one could potentially apply the nonresonant photoionization imaging technique to almost all atoms and ions to obtain number/column densities with good accuracy. We have already shown how this can be done using DLP spectroscopy. ${ }^{25}$ The down side is that the lower limit on column densities becomes more like $10^{15} \mathrm{~cm}^{-2}$ since even at threshold nonresonant cross sections rarely exceed $5 \mathrm{MBar}$ or so. We intend to explore this direction in future experiments.

On the instrumentation side we are currently upgrading to a high-resolution $1 \mathrm{~m}$ normal incidence system with a much brighter laser plasma continuum source. Higher VUV flux will permit better matching of photon bandwidth to resonance (natural) width which will in turn yield a broader absorbance dynamic range. We will also have both back thinned and gated CCD options for high sensitivity or better time resolution/sample plasma emission rejection, respectively. We also plan to collimate the VUV beam with a suitable toroidal mirror on the exit arm of the monochromator, which will simplify optical geometry and image analysis. Looking even further ahead we will explore the use of a grazing incidence monochromator based system to cover the $35-400 \mathrm{eV}$ photon energy range and also experiments with subnanosecond continuum light sources. ${ }^{26}$ This will help bridge the diagnostic gap between the present work and earlier work with crystal ( $\mathrm{keV}$ photons) dispersion and $\sim 100 \mathrm{ps}$ frametimes which required access to large laser facilities. ${ }^{27,28}$ 


\section{ACKNOWLEDGMENTS}

This work was funded by the European Union under the Training and Mobility of Researchers (TMR) Facilities Access Program. The DCU group acknowledges partial support from Enterprise-Ireland. The authors wish to thank Des Lavelle for the engineering of the target chambers and vacuum-optical coupling system.

${ }^{1}$ J. F. Ready, Effects of High Power Laser Radiation (Academic, New York, 1971).

${ }^{2}$ T. P. Hughes, Plasmas and Laser Light (Adam Hilger, London, 1975).

${ }^{3}$ S. Nakai and H. Takabe, Rep. Prog. Phys. 59, 1071 (1996).

${ }^{4}$ C. H. Skinner, Phys. Fluids B 3, 2420 (1991).

${ }^{5}$ J. Workman, A. Maksimchuk, X. Liu, U. Ellenberg, J. S. Coe, C. Y. Chien, and D. Umstadter, Phys. Rev. Lett. 75, 2324 (1995).

${ }^{6}$ I. C. E. Turcu et al., Proc. SPIE 2015, 243 (1993).

${ }^{7}$ O. E. Meighan et al., Appl. Phys. Lett. 70, 1497 (1997).

${ }^{8}$ Proceedings of the Fourth International Conference on Laser Ablation, edited by R. E. Russo, D. B. Geohegan, R. F. Haglund, Jr., and K. Murakami, Appl. Surf. Sci. 127-129 (1997).

${ }^{9}$ S. Amoruso, R. Bruzzese, N. Spinelli, and R. Velotta, J. Phys. B 32, R131 (1999).

${ }^{10}$ D. B. Geohegan, Appl. Phys. Lett. 60, 2732 (1992).

${ }^{11}$ W. W. Whitty and J-P. Mosnier, Appl. Surf. Sci. 127-129, 1035 (1998).

${ }^{12}$ G. W. Martin, L. A. Doyle, A. Al-Khateeb, I. Weaver, M. J. Lamb, T.
Morrow, and C. L. S Lewis, Appl. Surf. Sci. 129, 710 (1998).

${ }^{13}$ P. K. Carroll and E. T. Kennedy, Phys. Rev. Lett. 38, 1068 (1977).

${ }^{14}$ J. T. Costello, E. T. Kennedy, J-P. Mosnier, P. K. Carroll, and G. O'Sullivan, Phys. Scr., T 34, 77 (1991).

${ }^{15}$ E. Jannitti, M. Gaye, M. Mazzoni, P. Nicolosi, and P. Villoresi, Phys. Rev. A 5, 4033 (1993).

${ }^{16}$ P. K. Carroll, E. T. Kennedy, and G. O'Sullivan, Appl. Opt. 19, 1454 (1980).

${ }^{17}$ E. T. Kennedy, J. T. Costello, J-P. Mosnier, A. A. Caffola, M. Collins, L. Kiernan, U. Köble, M. H. Sayyad, and M. Shaw, Opt. Eng. 33, 3964 (1994).

${ }^{18}$ J. M. Bizau et al., Phys. Rev. Lett. 67, 576 (1991).

${ }^{19}$ I. C. Lyon, B. Peart, K. Dolder, and J. B. West, J. Phys. B 20, 1471 (1987).

${ }^{20}$ R. K. Singh and J. Narayan, Phys. Rev. B 41, 8843 (1990).

${ }^{21}$ D. Colombant and G. F. Tonon, J. Appl. Phys. 44, 3524 (1973).

${ }^{22}$ R. D. Cowan, The Theory of Atomic Structure and Spectra (University of California Press, Berkeley, CA, 1981).

${ }^{23}$ W. W. Whitty, Ph.D. thesis, DCU, 1998.

${ }^{24}$ A. Corney, Atomic and Laser Spectroscopy (Clarendon, Oxford, 1977).

${ }^{25}$ W. W. Whitty, J. T. Costello, E. T. Kennedy, C. Moloney, and J-P. Mosnier, Appl. Surf. Sci. 127-129, 686 (1998).

${ }^{26}$ O. E Meighan et al., Phys. Rev. A 40, 330 (1989).

${ }^{27}$ A. Lamb and J. T. Costello, Appl. Phys. Lett. 70, 1497 (1997).

${ }^{28}$ D. M. O'Neill, C. L. S. Lewis, D. Neely, S. J. Davidson, S. J. Rose, and R. W. Lee, Phys. Rev. A 44, 2641 (1991). 